\title{
Philosophiques
}

\section{Les fictions de la raison pure}

\section{Claude Piché}

Volume 13, numéro 2, automne 1986

URI : https://id.erudit.org/iderudit/203321ar

DOI : https://doi.org/10.7202/203321ar

Aller au sommaire du numéro

Éditeur(s)

Société de philosophie du Québec

ISSN

0316-2923 (imprimé)

1492-1391 (numérique)

Découvrir la revue

Citer cet article

Piché, C. (1986). Les fictions de la raison pure. Philosophiques, 13(2), 291-303.

https://doi.org/10.7202/203321ar

\section{Résumé de l'article}

On est porté à sous-estimer le rôle authentiquement productif de la raison dans la Dialectique transcendantale de la Critique de la raison pure. Cet article propose une illustration de ce rôle à l'occasion des Paralogismes de la raison pure, qui ne sont rendus possibles que sur la base d'une fiction négative opérée à partir du sens interne. Cette illustration s'inscrit dans la perspective plus large d'une interprétation de la Dialectique transcendantale en vertu d'une théorie du schématisme, laissée au second plan par Kant en raison de la prépondérance des motivations critiques.
Ce document est protégé par la loi sur le droit d'auteur. L'utilisation des services d’Érudit (y compris la reproduction) est assujettie à sa politique d'utilisation que vous pouvez consulter en ligne.

https://apropos.erudit.org/fr/usagers/politique-dutilisation/ 


\title{
LES FICTIONS DE LA RAISON PURE
}

\author{
par Claude Piché
}

\begin{abstract}
RÉSUME. On est porté à sous-estimer le rôle authentiquement productif de la raison dans la Dialectique transcendantale de la Critique de la raison pure. Cet article propose une illustration de ce rôle à l'occasion des Paralogismes de la raison pure, qui ne sont rendus possibles que sur la base d'une fiction négative opérée à partir du sens interne. Cette illustration s'inscrit dans la perspective plus large d'une interprétation de la Dialectique transcendantale en vertu d'une théorie du schématisme, laissée au second plan par Kant en raison de la prépondérance des motivations critiques.
\end{abstract}

ABSTRACT. We tend to underestimate the authentically productive role of reason in the Transcendental Dialectic of the Critique of Pure Reason. This paper suggests an illustration of this role as it can be traced in the Paralogisms of Pure Reason, which themselves became possible on the basis of a negative fiction exerted toward the inner sense. This illustration is part of a larger perspective aiming at an interpretation of the Transcendental Dialectic on the ground of a theory of schematism, which is somehow overshadowed by Kant's critical intentions.

L'un des principaux obstacles à la compréhension de la théorie des idées transcendantales de la Critique de la raison pure tient au fait que Kant ne nous éclaire véritablement sur la fonction régulatrice de celles-ci que dans l'«Appendice à la dialectique transcendantale», c'est-à-dire en fin de parcours. On apprend alors que l'idée de la raison, par-delà l'illusion transcendantale qu'elle provoque presque irrésistiblement, est en réalité un schème visant à faciliter l'unité systématique des connaissances empiriques. Jusqu'à quel point est-il permis de parler dans ce cas d'un schématisme de la raison pure ${ }^{1}$ qui' puisse faire écho au

1. Cf. de l'auteur « Le schématisme de la raison pure. Contribution au dossier HeideggerKant» dans Les études philosophiques, $N^{\circ} 1$, janvier-mars 1986, p. 79-99. 
schématisme de l'entendement introduit dans la première partie de la Logique transcendantale? Une réponse positive à cette question implique que nous soyons en mesure de localiser au sein de la Dialectique transcendantale une instance capable d'assumer un rôle équivalent à celui de l'imagination dans le schématisme des concepts de l'entendement. La tâche consiste en fait à déceler l'instance qui donne naissance à ces schèmes avant même que ceux-ci ne donnent prise à une utilisation fautive. Chose certaine, il ne peut s'agir à nouveau de l'imagination sensible puisque les idées de la raison transcendent principiellement le monde phénoménal. Si l'on veut mener à bien une telle lecture à rebours de la Dialectique transcendantale à la lumière d'une théorie du schématisme, il faut être en mesure de montrer comment la raison est elle-même une faculté productrice à l'instar de l'imagination sensible, même si dès lors la raison se voit contrainte de schématiser sans intuition, c'est-à-dire simplement à l'aide de concepts.

Kant ne nous facilite pas la tâche ici dans la mesure où dès l'introduction à la Dialectique transcendantale, il insiste surtout sur le fait que les idées de la raison pure sont issues de syllogismes. Or chacun sait qu'un syllogisme ne vise pas à établir de nouvelles connaissances et encore moins à produire de nouveaux objets, mais bien plutôt à déterminer, comme l'affirme Kant, une connaissance particulière en la subsumant sous un concept général. Le syllogisme en tant que tel n'est pas vraiment productif ; seul un syllogisme fallacieux peut prétendre engendrer une connaissance nouvelle, et par là faire naître une apparence transcendantale. C'est une subreption dans les prémisses de chacune des trois séries de syllogismes de la raison pure qui entraîne une dialectique de la raison, si bien qu'une fois l'erreur dissipée, on voit mal comment pourrait subsister un espace de jeu pour quelque chose de tel qu'un schématisme. A vrai dire, Kant insiste à tel point sur les motivations dogmatiques qui sont à l'origine de ces subreptions qu'il fait passer à l'arrière-plan les modalités précises du mécanisme d'engendrement de ces objets fictifs que sont les trois idées de la raison, laissant ainsi dans l'ombre sa théorie du schématisme de la raison, qui fait surface, de manière quelque peu inopinée, dans l'Appendice. Il importe pourtant de distinguer à l'occasion de chacune de ces trois idées transcendantales comment la raison 
engendre son schème avant même que celui-ci ne soit interprété dogmatiquement comme un objet réel. Cela exige un travail d'analyse complexe au niveau de la structure de chacune des trois idées, travail dont nous avons déjà livré certains résultats ${ }^{2}$, mais qu'il nous est impossible de reprendre dans les limites de cet article. Nous nous restreindrons ici à la première idée transcendantale, telle qu'elle apparaît dans les Paralogisme de la raison pure, dans le but non pas d'en faire l'étude exhaustive, mais plutôt de montrer simplement comment la raison entre en jeu comme faculté productrice, comme facultas figendi, pour reprendre le vocabulaire de la psychologie de l'époque. Certes, la démonstration du caractère schématisant de la raison est ici particulièrement délicate, plus encore que dans le cas des Antinomies et de l'Idéal transcendantal; mais par ailleurs le type d'intervention de la raison dans la psychologie rationnelle recèle des aspects très révélateurs quant à l'essence de cette faculté. Afin de simplifier la tâche au maximum, l'exposé sera axé sur l'analyse d'un court extrait, faisant suite à une mise au jour des prémisses du problème. Il s'agit donc dans un premier volet de préciser la nature de l'objet servant de point de départ à la psychologie rationnelle, à savoir le Je ou Je pense compris comme abstraction; un deuxième volet, plus ample cette fois, contribuera à mettre en lumière l'aspect productif de la raison conduisant à l'hypostase qui est à l'origine de l'illusion dans la psychologie rationnelle.

\section{LE JE: UNE ABSTRACTION}

Kant ne cesse d'insister sur l'indigence extraordinaire du point de départ de la psychologie rationnelle; son seul «texte», dit-il, réside dans l'unique pronom Je, ou encore dans la proposition Je pense. Il s'agit là, on le sait, du véhicule qui doit pouvoir accompagner toutes les représentations; le Je ne joue ici le rôle que de condition subjective de toute pensée. Il accompagne assurément toutes les représentations, mais le privilège de cette polyvalence est payé très cher, si l'on note incidemment que le Je, en tant que tel, est une « représentation simple et par elle-même

2. Cf. de l'auteur Das Ideal: Ein Problem der Kantischen Ideenlehre. Bouvier Verlag, Bonn, 1984. 
totalement vide de contenu» ${ }^{3}$. Le Je partage la richesse des données empiriques présentes dans la perception interne, et l'on ne peut avoir d'autre expérience de ce Je qu'en se tournant vers le sens interne, comme le seul lieu de sa manifestation qui nous soit accessible. Par suite, si l'on veut obtenir une représentation du Je pris pour lui-même, on doit s'en remettre à une abstraction à l'égard des multiples données empiriques du sens interne. Mais alors la représentation Je dans toute sa pureté n'est rien d'autre que la «plus pauvre de toutes les représentations » ${ }^{4}$. L'abstraction est portée ici à un niveau très élevé, et c'est ce qui fait que Kant hésite à qualifier le Je de «concept»" ${ }^{5}$ : sa représentation est complètement vide, il ne contient rien "en soi», quoique, si l'on considère l'ampleur et la richesse de l'expérience interne d'où il a été tiré par abstraction, on puisse affirmer qu'il contient beaucoup "sous lui» ${ }^{6}$. Au vide de contenu de la représentation Je correspond l'importance de son étendue, et lorsque Kant met en rapport et souligne à la page $\mathrm{A} 785$ le contenu ainsi que l'étendue du Je à l'aide des expressions «en soi» et «sous lui», c'est manifestement en songeant au principe logique bien connu selon lequel l'intension et l'extension d'un concept ont un rapport inversement proportionnel ${ }^{7}$. Le domaine d'application d'un concept croît proportionnellement à l'élimination des traits particuliers qui constituent le contenu du concept.

Le texte du chapitre sur les Paralogismes de la raison pure indique de façon très nette et à plusieurs reprises l'origine de cette représentation vide qu'est le Je, à savoir qu'il est issu d'un processus d'abstraction. Or il peut s'avérer utile, avant de passer à l'étude de l'illusion comme telle, de dire un mot de la nature du

3. Kritik der reinen Vernunft (ci-après $\mathrm{KrV}$ ), A 345-346 (souligné par C.P.); nos citations de la Critique de la raison pure sont tirées de la traduction de Delamarre et Marty dans la "Bibliothèque de la Pléiade»: E. KANT, CEuvres pbilosophiques, Gallimard, Paris, 1980. Cette édition comporte également la pagination originale, seule utilisée ici.

4. $\mathrm{KrV}, \mathrm{B} 408$.

5. KrV, A 346; au mot « concept » (Begriff) pris dans son sens fort, Kant préfère dans les Prolegomena \46 le mot « désignation» (Bezeichnung) (trad. franç. parJ. GIBELIN : Prolégomènes, J. Vrin, Paris, 1968). Voir également KrV, A 382, ainsi que A 728 pour le caractère précaire de la « désignation».

6. $\mathrm{KrV}, \mathrm{A} 785$; Cf. A 355 .

7. Logik, éd. Jäsche, $\$ 7$; trad. franç. par L. Guillermit : Logique, J. Vrin, Paris, 1970. 
processus qui a rendu possible l'édification d'une telle représentation. Dans la Logique éditée par Jäsche $(\mathbb{S} 6)$, le thème de l'abstraction est introduit dans le cadre de la théorie de la formation des concepts. L'abstraction apparaît, aux côtés de la comparaison et de la réflexion, comme l'une des trois « opérations logiques » visant à construire des concepts : c'est-à-dire des représentations générales valables pour plusieurs individus. Cela signifie du même coup que la représentation Je est obtenue de manière analogue à celle qui permet d'obtenir n'importe quel concept général tiré de l'expérience, en l'occurrence ici: l'expérience interne. Le Je est une représentation abstraite au sens propre, et on doit même avouer qu'il correspond au point ultime auquel peut atteindre le processus d'abstraction, puisque la Critique de la raison pure identifie le Je pense à un simple "quelque chose» ${ }^{8}$, expression que Kant utilise dans le passage déjà mentionné de sa Logique ${ }^{9}$ pour désigner le concept le plus général qui soit. Il nous reste donc maintenant à nous interroger sur le destin de cette prétention de la psychologie rationnelle à tenir un discours légitime en se fondant sur un concept totalement vide, constituant le point le plus haut de l'opération d'abstraction.

\section{LIEU DE L'APPARENCE: UNE HYPOSTASE}

Déjà le titre «Paralogismes de la raison pure» fait mention dans une première approximation du lieu où l'illusion transcendantale prend place pour la psychologie rationnelle: nous avons affaire à un raisonnement spécieux. Mais si l'on y regarde de plus près, on apprend que la nature précise de la faute commise dans le raisonnement consiste en une bypostase: en effet, l'apparence transcendantale au niveau des Paralogismes réside dans une "subreption de la conscience hypostasiée » ${ }^{10}$. Passons d'abord en revue les éléments qui composent cette expression: la subreption, il va sans dire, fait référence au vice qui consiste à obtenir quelque chose par des moyens détournés, de manière illicite. L'hypostase par ailleurs est clairement définie à l'intérieur même de notre

\footnotetext{
8. $\mathrm{KrV}, \mathrm{A} 355$.

9. Logik, éd. Jäsche, $\$ 6$.

10. $\mathrm{KrV}, \mathrm{A} 402$.
} 
chapitre: il s'agit de la transposition de ce qui dans notre esprit se trouve à l'état de simple "représentation» en un "objet»" 11 existant qui subsiste indépendamment de notre représentation. On hypostasie lorsqu'on interprète ses propres « pensées » comme des «choses» réelles ${ }^{12}$. Or, puisque l'hypostase dans les Paralogismes porte sur la conscience, cela signifie simplement que l'apparence tient ici au fait que l'on saisit cette conscience, qui n'est rien de plus qu'une condition logique de la pensée, comme un objet. Ainsi avec Kant on peut dire dans ce cas que «toute apparence consiste à prendre la condition subjective de la pensée pour la connaissance de l'objet» ${ }^{13}$. Kant souligne ici les mots «subjectives» et «objet» pour marquer la transition entre sujet et objet à laquelle on assiste dans l'hypostase de la psychologie rationnelle. C'est également sur le caractère subreptice de cette transition que Kant insiste lorsqu'il affirme que le sujet transcendantal en tant que pôle déterminant ne peut impunément passer au pôle déterminé, c'est-à-dire celui de l'objet. Nous savons désormais que ce Je n'est rien d'autre qu'une représentation vide, si bien que ce qui peut se dire de cette simple représentation subjective ne vaut pas automatiquement comme la connaissance d'un objet: "mais la simplicité de la représentation d'un sujet n'est pas pour cela une connaissance de la simplicité $d u$ sujet lui-même ${ }^{14}$. On assiste ici au passage inavoué entre la simple représentation et la chose elle-même qui est présenté comme étant la cause de toute l'illusion dans les Paralogismes.

Les remarques qui précèdent nous ont permis de nous rappeler les lignes de force bien connues qui animent tout le chapitre sur les Paralogismes. Toutefois, si nous avons pu identifier les deux pôles entre lesquels l'hypostase opère un passage, nous demeurons dans l'ignorance totale quant à la modalité de cette assimilation. C'est la raison pour laquelle nous aimerions attirer l'attention sur un extrait d'une importance capitale tiré de la version remaniée de notre chapitre qu'offre la deuxième édition de la Critique de la raison pure. Voici donc l'extrait en question:

11. $\mathrm{KrV}, \mathrm{A} 395$.

12. $\mathrm{KrV}, \mathrm{A} 395$.

13. $\mathrm{KrV}, \mathrm{A} 396$.

14. $\mathrm{KrV}, \mathrm{A} 355$ (souligné par C.P.). 
L'apparence dialectique dans la psychologie rationnelle vient de ce que l'on confond une idée de la raison (l'idée d'une intelligence pure) avec le concept indéterminé à tous égards d'un être pensant en général. Je me pense moi-même en vue d'une expérience possible, en faisant abstraction de toute expérience réelle, et j'en conclus que je puis avoir conscience de mon existence même en dehors de l'expérience et des conditions empiriques de celle-ci.

Je confonds donc l'abstraction possible de mon existence empiriquement déterminée avec la prétendue conscience d'une existence possible du moi pensant à l'état isolé, et je m'imagine connaître ce qu'il y a en moi de substantiel comme le sujet transcendantal, tandis que je n'ai dans la pensée que l'unité de la conscience qui est au fondement de tout acte de détermination considéré comme simple forme de la connaissance ${ }^{15}$.

La première phrase insiste sur le fait que le «concept indéterminé» d'un être pensant est confondu avec une idée de la raison: donc encore ici il est question de l'hypostase, mais cette fois Kant emploie le mot Verwechslung (confusion) pour signaler ce qui a lieu en vérité à l'occasion de celle-ci. On assiste en fait avec la Verwechslung à un glissement dans le sens des termes, et c'est précisément ce trait qui est visé lorsque Kant par ailleurs désigne la faute des Paralogismes de la psychologie comme un sophisma figurae dictionis ${ }^{16}$ : il s'agit ici à vrai dire d'un déplacement de sens dans l'usage d'un même mot, à savoir le terminus medius.

C'est cependant la dernière phrase de l'extrait qui recèle la clé de l'explication de la Verwechslung. Kant retrace le sens du

15. $\mathrm{KrV}, \mathrm{B}$ 426-427. La première édition de la $\mathrm{KrV}$ comporte également des passages dans lesquels l'opération d'abstraction est considérée comme insuffisante pour justifier l'accès à des objets transcendants par rapport à l'expérience. En fait, Kant affirme qu'une représentation simple obtenue par abstraction est loin d'équivaloir à la connaissance d'un objet simple en tant que tel. Pour atteindre ce dernier, la raison dogmatique doit faire, comme nous allons le voir, un pas de plus. Reprenons de manière plus exhaustive le passage déjà cité à la note 14 ( $\mathrm{KrV}$, A 355): «Mais la simplicité de la représentation d'un sujet n'est pas pour cela une connaissance de la simplicité du sujet lui-même, car nous faisons tout à fait abstraction de ses propriétés, quand nous le désignons simplement par cette expression totalement vide de contenu : Je (expression que je puis appliquer à tout sujet pensant).» Voir également $\mathrm{KrV}, \mathrm{A} 785$ : «Or, par là même que le simple dans l'abstraction est tout à fait distinct du simple dans l'objet, et que le Je, qui dans le premier sens ne renferme en soi aucune diversité, peut être, dans le second, où il signifie l'âme même, un concept très complexe, c'est-à-dire contenir et désigner beaucoup de choses sous lui, je découvre un paralogisme.» (souligné par Kant)

16. $\mathrm{KrV}, \mathrm{B} 411$, A 402 ; cf. Logik, éd. Jäsche, $\$ 90$. 
déplacement opéré en faisant ressortir dans le texte par un soulignement les mots abstraction et isolé: il s'agit en fait du passage du moi pensant abstrait à l'existence du moi pensant à l'état isolé. Or il est possible pour le lecteur du texte allemand de succomber à une méprise dans la mesure où les termes Abstraktion et abgesondert soulignés par Kant pour marquer l'opposition sont très souvent utilisés indifféremment l'un pour l'autre ; ce qui donnerait une allure quasi tautologique au déplacement de sens. En effet, le mot Absonderung constitue l'équivalent allemand du latin abstractio: Meier par exemple dans son Auszug aus der Vernunftlebre ${ }^{17}$ utilise la logische Absonderung pour traduire l'abstractio logica. De même, Kant dans son traité de 1764 sur l'Évidence des principes de la théologie naturelle et de la morale caractérise les concepts abstraits, qui sont le propre de la philosophie, par l'expression $A b s o n d e r u n g{ }^{18}$; on retrouve également cette utilisation synonymique d'Abstraktion et d'Absonderung dans la Logique éditée par Jäsche $(\$ 6)$.

Pourtant, et ceci constitue le point central de notre argumentation, la juste saisie de la solution présentée par Kant dans l'extrait cité réclame que nous nous abstenions de comprendre Abstraktion et Absonderung comme des expressions synonymes. Dans tout le chapitre sur les Paralogismes ${ }^{19}$, l'utilisation du participe passé abgesondert ne peut pas être assimilée au processus de l'abstraction ${ }^{20}$ décrit ci-dessus. Il nous incombe alors d'indiquer la portée de l'hypostase en question en tant qu'elle aboutit à poser «l'existence possible du moi pensant à l'état isolé». Les expressions «isolé» et «isolément» par lesquelles les traducteurs rendent fort justement le participe abgesondert renvoient en fait à quelque chose de tout autre qu'au processus formel de l'abstraction. Car à côté des concepts que l'on obtient par ce processus "logique», il y a une autre façon d'obtenir des concepts qui

17. George Friedrich MEIER, Auszug aus der Vernunftlebre, Gebauer, Halle, $1752, \$ 259$; ce texte est reproduit dans l'édition de l'Akademie des Gesammelte Schriften (ciaprès G.S.) de Kant, tome XVI, p. 549-550.

18. Untersuchung über die Deutlichkeit der Grundsätze der natürlichen Theologie und der Moral, G.S., t. II, p. 276; trad. franç. par J. FerRaRI, «La Pléiade», p. 216. Voir aussi les «Réflexions» de Kant selon la numérotation de l'édition de l'Akademie $: \$ 2907$, $2847,2858$.

19. $\mathrm{KrV}$, A 346, B $410,419,420,430$.

20. Les traducteurs français ne se plient pas toujours à cette exigence, ainsi Tremesaygues et Pacaud (P.U.F.), p. 301, et à leur suite Delamarre et Marty («La Pléiade»), p. 1060. 
consiste à les fabriquer de toutes pièces (conceptus factitii ${ }^{21}$ ). Ici entre en jeu une faculté active d'un type bien précis, que la psychologie empirique classique nomme facultas figendi ${ }^{22}$ : donc la faculté d'ériger des fictions, et en l'occurrence d'inventer des concepts. On songe immédiatement à l'exemple du centaure, issu de la combinaison d'un corps de cheval et d'un corps d'homme. Mais la faculté d'inventer peut aussi opérer de façon privative; le processus est alors décrit par Wolff non plus comme compositio mais comme divisio ${ }^{23}$ ou encore comme separatio ${ }^{24}$. De même Baumgarten dira que la facultas figendi fonctionne tout aussi bien praescindendo que combinando ${ }^{25}$. Ce qu'il est important de retenir ici, c'est que la faculté d'inventer en général peut procéder de façon tout aussi bien négative, en retranchant à certaines représentations des traits qui leur appartiennent pourtant en propre, que de façon positive en assemblant des éléments disparates. Et c'est dans ce cadre précis que vient s'inscrire le participe abgesondert dans notre extrait.

Quant à la question de savoir si Kant connaissant ces deux fonctions distinctes de la faculté d'inventer, qu'il suffise de rappeler que Kant s'est servi de la Métaphysique de Baumgarten comme manuel pendant toute sa carrière de professeur. Il ne sera donc pas étonnant de constater que déjà très tôt dans les leçons sur la métaphysique qui nous sont parvenues, la fonction privative (praescindendo) de la faculté de fabriquer des concepts est mentionnée, et à vrai dire précisément en rapport avec la genèse du concept d'« esprit» par opposition à celui d'âme humaine ${ }^{26}$ !

21. Logik, éd. Jäsche, $\$ 4$; cf. Réfl. $\$ \$ 2852,2862$.

22. Cf. Christian WOLfF, Psychologia empirica, $\$ 144$, in Gesammelte Werke, Olms, Hildesheim, 1968, Sect. II, T. 5, p. 97. Voir également Alexander Gottlieb BAUMGARTEN, Metaphysica \$589, Hemmerde, Magdebourg, 1757; texte reproduit dans les G.S. de Kant, t. XV, p. 26.

23. Psychologia empirica, $\$ 144$.

24. Ibid., $\$ 139$.

25. Metaphysica, $\$ 589$.

26. «La faculté d'inventer [Dichtungsvermögen] : - elle rassemble des concepts d'une manière différente de ce que l'on retrouverait dans l'imagination [Phantasie]. 1) combinando: par exemple des hommes avec des ailes, par exemple Mercure, Pégase -2) praescindendo: ce mode doit être distingué du mode abstrabendo [et ici c'est Kant qui souligne! C.P.], dans la mesure où ce dernier veut dire: ne pas penser quelque chose: laisser de côté un concept à propos de quelque chose; et le premier mode [signifie] : nier quelque chose, abolir le trait de pensée. — [ainsi dans 
D'ailleurs les leçons sur la métaphysique éditées par Pölitz, à peu près contemporaines de la rédaction de la Critique de la raison pure, insisteront à nouveau sur l'intervention de la séparation (separieren) à l'occasion de l'édification d'une pneumatologie ${ }^{27}$.

Quoi qu'il en soit, ce qui importe pour l'instant, ce sont les conséquences, pour la compréhension de notre extrait, de l'interprétation de la subreption comme étant attribuable à une activité créatrice de la raison, saisie comme facultas figendi. Le passage du moi pensant abstrait au moi pensant à l'état isolé ne signifie plus le passage du même au même, c'est-à-dire de l'abstraction à l'abstraction. Il s'agit bien plutôt du passage de cette opération logique purement formelle qu'est l'abstraction à une opération pour ainsi dire transcendantale et réelle visant à construire un concept (celui d'un pur esprit, désincarné), dont on ignore s'il a un objet. Si l'obtention d'un concept général à l'aide de l'abstraction comporte un aspect négatif dans la mesure où certains traits particuliers appartenant toujours en propre à l'objet sont laissés momentanément dans l'ombre au profit de la généralité du concept ainsi obtenu, l'aspect inoffensif de cette opération négative n'a pas de commune mesure avec la violence de la coupure imposée à l'objet lui-même lors de la construction d'un concept

le mode abstrabendo, C.P.] je laisse de côté un prédicat de ma fantaisie [Phantasie] dans une abstraction je ne pense qu'un concept partiel - si cependant je nie les autres concepts partiels, alors je commence déjà à inventer [fingieren]. Toutes les inventions [Erdichtungen] sont novatrices: et à vrai dire de manière simplement partielle dans les perceptions sensibles. Elles sont: 1) intellectuelles: ainsi par exemple en philosophie : le concept d'esprit a été arbitrairement abstrait du concept d'air par séparation et combinaison.» Vorlesungen über Metaphysik (Herder), Loses Blatt XXV, 38, in G.S., t. XXVIII, 1, p. 143. Voir également le passage suivant : «Facultas fingendi. Produire des représentations qui ne [sont] pas des sensations présentes ou passées. Cela se réalise 1) combinando, quae in sensationibus non erant combinata 2) sejungendo, quae in sensationibus non erant sejuncta. Les abstractions ne sont pas novatrices (sinon formaliter: d'abord confusément et ensuite distinctement), mais la facultas figendi est novatrice, par exemple Cerbère : chien qui réunit deux têtes. La facultas fingendi est novatrice aussi separando [...] La faculté d'inventer [Dichtungsvermögen] est plus générale que ce que l'on entend normalement sous le vocable "poête» [Dichter], ainsi en mathématique et en philosophie toutes les définitions arbitraires - Les hypothèses sont [issues] de la faculté d'inventer intellectuelle: au sens courant des conceptus ficti. Par exemple, l'esprit n'est pas un concept d'expérience pas plus qu'un concept abstrait.» Ibid., G.S., t. XXVIII, 2, p. 860. De même, voir Vorlesungen über Logik (Blomberg), G.S., t. XXIV, p. 261-263.

27. Vorlesungen über Metaphysik (Pölitz), G.S., t. XXVIII, 1, p. 277-278; voir également l'utilisation de Absondern au sens de Trennen in ibid., p. 273-274. 
fictif ${ }^{28}$. Kant parle dans ce cas de «synthèse négative» ${ }^{29}$ au sens où la négation comme processus réel atteint l'objet lui-même, à savoir ici la séparation du Je à l'égard de l'expérience empirique du sens interne, où il peut seul se manifester. Le passage de l'abstraction formelle à la séparation réelle dans le cas du Je signifie que l'on passe de la représentation vide du sujet transcendantal à la considération de l'existence de cette simple représentation à titre d'objet réel. A vrai dire, il est tout aussi illégitime de prétendre connaître la réalité du Je pense lorsque coupé de son rapport essentiel au sens interne, qu'il serait absurde par exemple de postuler pour le concept vague et général d' «arbre» l'existence d'un objet correspondant exactement et uniquement à la généralité du concept, sans toutefois être spécifié comme individu sous la forme par exemple d'un « pin», d'un « saule» ou d'un «tilleul» ${ }^{30}$; toutes espèces dont le concept empirique d'arbre est pourtant tributaire. La séparation, qu'elle prenne place entre la représentation générale d'un arbre et ses diverses instances particulières ou entre le Je et les représentations concrètes qu'il doit toujours accompagner, ne peut dans les deux cas que conduire à des entités hautement «problématiques», au sens kantien du terme ${ }^{31}$.

Un traitement plus exhaustif du problème exigerait également que nous nous attardions plus longuement sur l'utilisation très symptomatique chez Kant de l'expression Substantiale (dans notre extrait: le «substantiel») pour désigner l'aspect privatif du statut du Je. En effet, ce terme technique latin ${ }^{32}$ employé par Kant désigne «ce qui demeure» de la substance «quand tous les accidents ont été éliminés (abgesondert!)» ${ }^{33}$. Quand on pense

28. Dans les trois passages des «leçons» auxquels nous faisions allusion à la note 24 , et où Kant montre que le concept d' "esprit» est issu de la faculté d'inventer, Kant prend bien soin d'indiquer, et ce dans chacun des trois passages (!), la différence fondamentale qui existe entre ces deux genres de négation. Il suit en cela l'exemple de Meier, cette fois-ci dans sa "grande» Vernunftlebre de 1752 (\$292, p. 426-428), qui distingue nettement entre $A b$ sonderung «logique» et une autre produite par un acte de l'« imagination» (Einbildungskraft): en effet, celle-ci atteint la «chose même» (Sache selbst). On retrouve des extraits du paragraphe 292 dans les notes d'Adikes au tome XVI des G.S., p. 549-551.

29. Réfl. $\$ 2907$.

30. Logik, éd. Jäsche, $\$ 6$.

31. $\mathrm{KrV}, \mathrm{A} 347$.

32. Baumgarten, Metaphysica, $\$ 196$.

33. Prolegomena, $\$ 46$. Cf. $\mathrm{KrV}, \mathrm{A} 414$. 
que tous les accidents de l'âme ne peuvent être que sensibles, on se rend compte de l'importance de la césure imposée par la fiction du Je lorsque celui-ci est réduit, pour satisfaire les ambitions de la psychologie rationnelle, à l'état de Substantiale.

Le bilan de notre analyse se lit comme suit : l'hypostase de la conscience constitue la forme propre de l'illusion transcendantale dans la psychologie rationnelle; elle consiste, comme nous l'avons vu, dans la confusion du Je pense comme condition subjective de la pensée avec ce même Je pense conçu cette fois comme objet. Il s'agit en fait du passage de la représentation abstraite Je à un objet réel. Nous avons tenté de mettre en lumière non pas tant les termes de ce rapport que l'explication du mécanisme qui permet le glissement subreptice et inconscient (puisqu'il s'agit d'un paralogisme ${ }^{34}$ ), de l'un dans l'autre. Tout le problème nous a paru résider dans l'assimilation illégitime, au niveau de la mineure du syllogisme, de deux processus de formation des concepts diamétralement opposés : l'abstraction et la fiction négative. En montrant que l'objet de la psychologie rationnelle est construit, en dépit de toute prétention à la rigueur, grâce à une activité fabulatrice de la raison, Kant est très tôt amené à considérer ce type de discours métaphysique sur le même pied que la pneumatologie de Swedenborg, c'est-à-dire au niveau de l'arbitraire du "rêve» ${ }^{35}$. A la suite de cette mise au point, le seul discours scientifique permissible sur la nature spirituelle de l'homme doit être nécessairement ramené au plan empirique, c'est-à-dire à l'âme, comme objet du sens interne. Celle-ci n'est par conséquent susceptible que d'une psychologie empirique, encore que Kant manifeste des réticences ${ }^{36}$ quant à la scientificité d'un tel discours. Si toutefois on renonce à toute prétention directement cognitive, il est possible d'édifier un

34. Logik, éd. Jäsche, $\$ 90$.

35. Allusion au titre du fameux essai de 1765: Träume eines Geistersehers, erläutert durch Träume der Metaphysik; trad. franç. par B. LORTHOLARY pour «La Pléiade» : Rêves d'un visionnaire expliqués par des rêves métaphysiques. Voir à ce propos le passage très significatif où Kant prétend que la raison, si elle veut édifier une " pneumatologie», doit s'en remettre à une "fiction", qui elle-même a recours à des " négations», G.S., t. II, p. 251-252; trad. franç., p. 567.

36. KrV, A 381, 848-849. Cf. Metaphysische Anfangsgründe der Naturwissenschaft, Préface, éd. orig., p. X-XI; trad. franç. par J. GiBeLIN : Premiers principes métaphysiques de la science de la nature, J. Vrin, Paris, 1971. 
concept transcendant portant sur la spiritualité de l'âme humaine dans le but strictement de favoriser l'unité systématique de notre connaissance de l'expérience interne. Par là, la raison se dote d'un schème qui est issu de sa propre activité productrice. Les trois idées de la raison sont au sens propre des objets de raison, des entia rationis, répondant à une fonction régulatrice. En ce sens elles sont légitimes, c'est-à-dire correctement construites et employées à bon escient. C'est seulement l'hypos tase de ces schèmes, pour en faire des objets réels, qui fait que l'on passe d'entia rationis ratiocinatae à des entia rationis ratiocinantis. Tout dépend de l'utilisation que fait la raison de sa faculté productrice. Du côté des études kantiennes, beaucoup de travail demande encore à être accompli en vue de l'élucidation du schématisme propre à la raison pure. Chose certaine, il n'est désormais plus possible de donner à certaines expressions comme dichten, Erdichtung, das bloss Gedichtete (inventer, invention, ce qui est simplement inventé) qui parsèment la Dialectique transcendantale, un sens simplement métaphorique.

Département de philosophie

Université de Montréal 